\title{
Regional White Matter Atrophy Correlates with Spike Activity in Encephalopathy Related to Status Epilepticus During Slow Sleep (ESES) After Early Thalamic Lesions
}

\author{
Ana R. Oliveira ${ }^{1}$ - $\cdot$ Rita G. Nunes $^{1} \cdot$ Patrícia Figueiredo $^{1} \cdot$ Ana I. Dias $^{2} \cdot$ Alberto Leal $^{2,3}$
}

Received: 1 October 2019 / Accepted: 28 June 2020 / Published online: 11 July 2020

(c) Springer Science+Business Media, LLC, part of Springer Nature 2020

\begin{abstract}
Encephalopathy related to Status Epilepticus during slow Sleep (ESES) is an age-related, epileptic syndrome, which associates cognitive/behavioral disturbances with a peculiar pattern of spike activity. One promising line of research is the study of ESES in cases of early thalamic lesions. We studied 7 ESES patients with unilateral thalamic lesions using magnetic resonance imaging to assess regional white matter (WM) and thalamic nuclei volume differences, and long-term electroencephalogram recordings to localize the epileptogenic cortex. N170 event-related potentials were used to demonstrate the dysfunctional character of the WM abnormalities. Diffusion-weighted images in a subset of 4 patients were used to parcellate the thalamus and evaluate volume asymmetries, based on cortical connectivity. Large WM regional atrophy in the hemisphere with the thalamic lesion was associated with both cortical dysfunction and epileptic activity. A correlation was demonstrated between lesions in the pulvinar and the mediodorsal thalamic nuclei and WM atrophy of the corresponding cortical projection areas. We propose that these abnormalities are due to the widespread structural disconnection produced by the thalamic lesions associated to a yet unknown age-dependent factor. Further exploration of WM regional atrophy association with the spike activity in other etiologies could lend support to the cortical disconnection role in ESES genesis.
\end{abstract}

Keywords ESES syndrome $\cdot$ CSWS $\cdot$ Thalamic lesion $\cdot$ White matter $\cdot$ Thalamo-cortical disruption

$\begin{array}{ll}\text { Abbreviations } \\ \text { ESES } & \begin{array}{l}\text { Encephalopathy related to Status Epilepticus } \\ \text { during slow Sleep }\end{array} \\ & \text { EEG } \\ \text { DWI } & \text { Diffusion-weighted imaging } \\ \text { GFP } & \text { Global Field Power }\end{array}$

Handling Editor: Micah M. Murray.

Electronic supplementary material The online version of this article (https://doi.org/10.1007/s10548-020-00784-3) contains supplementary material, which is available to authorized users.

Ana R. Oliveira

a.rita.v.oliveira@tecnico.ulisboa.pt

1 ISR-Lisboa/LARSyS and Department of Bioengineering, Instituto Superior Técnico, Universidade de Lisboa, Lisbon, Portugal

2 Department of Pediatric Neurology, Hospital Dona Estefânia, Lisbon, Portugal

3 Department of Clinical Neurophysiology, Hospital Júlio de Matos, Lisbon, Portugal

$\begin{array}{ll}\text { sLORETA } & \text { Standard low-resolution tomography } \\ \text { Pulv } & \text { Pulvinar thalamic nucleus } \\ \text { MD } & \text { Mediodorsal thalamic nucleus } \\ \text { WM } & \text { White matter } \\ \text { O-T } & \text { Occipital-temporal }\end{array}$

\section{Introduction}

Encephalopathy related to Status Epilepticus during slow Sleep (ESES) is an age-related epileptic syndrome, characterized by a specific electroencephalogram (EEG) pattern of very abundant spike-wave discharges during non-rapid eye movement (non-REM) sleep, reducing significantly in REM sleep and wakefulness, and by associated neurocognitive regression (Tassinari and Rubboli 2019). It is currently the most common, treatable epileptic encephalopathy, and it is an important problem in pediatric neurology because although following spontaneous ESES resolution improvement of those cognitive dysfunctions generally occurs, in most patients some level of impairment remains (Dorris et al. 2019). 
Despite significant efforts dedicated to understanding the peculiar features of the syndrome, the responsible mechanism has eluded investigators. One of the main problems seems to be the wide number of different etiologies (Sánchez Fernández et al. 2012a) which makes it particularly difficult to find a common factor. One of these etiologies is neonatal thalamic lesions, generally associated with hemorrhage, which has attracted the attention of researchers since the first description of its association with ESES (Monteiro et al. 2001). It is worth noting that this association had also been previously reported by Incorpora et al. 1999 in one infant with primary unilateral thalamic hemorrhage after the neonatal period. Several studies have been published of cases of ESES with thalamic lesions (Guzzetta et al. 2005; Kelemen et al. 2006; Losito et al. 2015; Monteiro et al. 2001), and also on the high probability that patients with neonatal thalamic lesion have of developing ESES (Kersbergen et al. 2013; van den Munckhof et al. 2020). Building on previous knowledge and on original observations of nine patients (Leal et al. 2018), we proposed a model for ESES whereby the selective thalamo-cortical disconnection sets in motion a chain of events leading to the peculiar relationship of spike activity with the non-REM sleep stage. Although several components of the model have yet to be independently validated, the central role of the thalamo-cortical structural disconnection deserves a more detailed study as it can be present in other etiologies of ESES without thalamic lesions (Fernandez-Miranda et al. 2012).

The present study was motivated by the hypothesis that the origin of the epileptic events in ESES thalamic lesioned patients may lay on a partial disconnection between the thalamus and the cortex. Here, we quantify the changes in white matter (WM) associated with the thalamic lesion and correlate them with the sources of spike activity, in a group of patients with ESES and early thalamic lesions. For this purpose, we pursued three main objectives: (1) to assess whether the atrophy of the thalamic nuclei, mainly the pulvinar (Pulv) and the mediodorsal (MD) nuclei, was consistent with the WM changes using masks of the thalamic nuclei manually drawn on the structural images and, whenever possible, obtained according to the connectivity profile of the thalamus using diffusion-weighted imaging (DWI) scans; (2) to determine whether the WM atrophy was associated with neural dysfunction, as measured with the N170 potential; and (3) to correlate the epileptic activity distribution with the WM atrophy.

\section{Materials and Methods}

\section{Clinical and Cognitive/Behavioral Data}

A group of 7 patients (P1-P7) was selected from a population of children with the diagnosis of ESES (Leal et al.
2018), based on a history of cognitive and/or behavioral disturbance associated with an important increase in EEG spike activity at sleep onset (Spike Index above $85 \%$ in a whole-night recording) (Carvalho et al. 2020). All had clinical and imaging evidence of a unilateral thalamic lesion in the neonatal (P1, P3, P4, and P7) or prenatal period (P2, P5, and P6) (Table 1).

P1 suffered a right thalamus hemorrhage at day 18 of an otherwise normal neonatal period. He recovered in the following months and reached normal developmental milestones for walking and speech (Table 1). Epilepsy started at the age of 7 months but only at 3 years did a significant regression in cognitive skills (prominently language) become apparent. At the time of the current study (9 years), he was unable to complete neuropsychological assessment batteries designed for children above an age of 5 years, and the behavioral assessment revealed communication, autonomy, and socialization skills of a 3, 7 and 6 years old child, respectively.

P2's gestation was complicated by maternal preeclampsia but presented no obvious clinical problems in the neonatal period. Following the onset of epilepsy at the age of 2 years, a cognitive regression mainly in visual and visual-spatial domains became apparent, with prominent spatial disorientation. The brain MRI demonstrated a right thalamus infarct bordered by hemosiderin, supporting a pre-natal hemorrhagic stroke as the underlying etiology. Shortly before the time of assessment for the study (8 years), her IQ was in the inferior range (Table 1) and school performance was poor.

P3 presented a left thalamus hemorrhage at day 2 of life, from which she recovered to reach normal developmental milestones for walking and speech acquisition (Table 1). Following epilepsy onset at age 5 years, a regression in learning skills became apparent with difficulties in understanding more complex concepts. An assessment before the current study ( 8 years) revealed a borderline IQ, with a clearly pathological performance.

P4 suffered a right thalamus hemorrhage at day 2 of life from which she recovered very well, reaching normal developmental milestones at the appropriate age. A subtle residual left side hemiparesis persisted throughout. A global cognitive developmental assessment at the age of 2 years was normal (Table 1). Up to the time of the current study (4 years), no cognitive or behavioral problems became apparent.

P5 had an uneventful gestation and neonatal period, with normal acquisition of walking and speech. At the age of 2 years, the patient started with occasional partial seizures. MRI demonstrated a right thalamic atrophy bordered with hemosiderin, suggesting a previous hemorrhagic stroke. At the age of 7 years, the cognitive assessment revealed an IQ of 60. At the age of assessment for the current study (8 years), the neurological examination was normal, but behavioral disturbances with attentional deficit, agitation, 


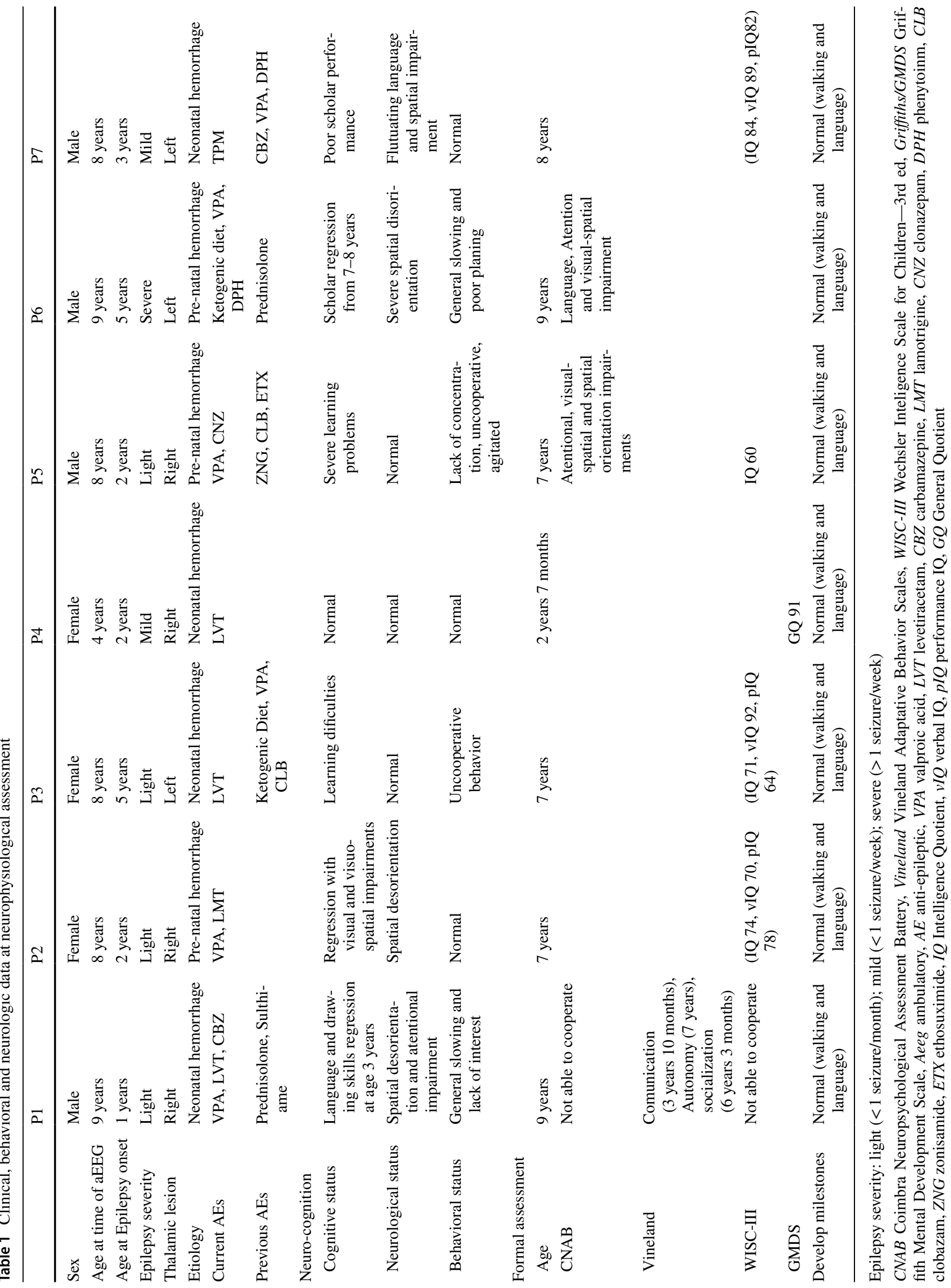


and severe learning problems ("he can read but does not understand the meaning") were prominent (Table 1). The parents report the onset of cognitive/behavioral problems at age 5 years.

P6 had an uneventful gestation and neonatal period with normal development up to the age of 3 years when epilepsy became apparent soon followed by a significant loss of verbal expression and drawing skills. Spatial disorientation was also a prominent behavioral disturbance. A brain MRI revealed an old left thalamus infarct. The assessment at the age of the present study ( 9 years) with the CNAB (Coimbra Neuropsychological Assessment Battery (Lopes et al. 2014), a tool validated for the Portuguese population age 5-15 years) revealed language impairment in memory, receptive and expressive components, as well as attentional deficits, but good executive functions (Table 1).

P7 suffered a left thalamus hemorrhage at day 33 of life from which he recovered to reach the normal milestones for the acquisition of walking and verbal expression. Epilepsy onset was at the age of 3 years and soon afterward problems were reported in spatial orientation, with persistent difficulties to follow appropriate routes in his neighborhood. A formal assessment at the age of study (8 years) revealed a normal IQ (Table 1).

The study was approved by the local ethics committee, and all data was collected in the context of clinical investigation and treatment.

\section{MRI Data}

\section{Structural Volumetry}

In all patients, a structural whole-brain MRI scan was obtained using an MPRAGE or an IR-FSPGR sequence with $1 \mathrm{~mm}^{3}$ voxel size. Imaging was performed on a $3 \mathrm{~T}$ Siemens Verio scanner for P1 and P2, on a 1.5 T GE Signa scanner for P3, P4, P6 and P7, and on a 3 T GE Signa scanner for P5.

A reorientation of the brain volume to the intercommissural reference (AC-PC) was performed, followed by manual segmentation of the thalamus, as well as the Pulv and MD nuclei, following the rules described in Power et al. 2015 and Byne et al. 2001, respectively, using MRICron (University of South Carolina) to delineate the masks. These two thalamic nuclei were chosen due to the fact that they are the ones with the largest volumes and are also affected in most of the patients studied. The relative atrophy of each nucleus was estimated, by taking as reference the volume of the contralateral nucleus in the hemisphere without the thalamic lesion $\left(1-\mathrm{Vol}_{\text {Lesioned }} / \mathrm{Vol}_{\text {Healthy }}\right)$.

In order to assess the existence of regional WM atrophy in the hemisphere with the thalamic lesion, the Freesurfer 6.0 software (Schiffler et al. 2017) was used to automatically segment the WM into 34 areas per hemisphere of the
Desikan-Killiany atlas (Desikan et al. 2006). Special care was taken to deal with the enlarged ventricles of the patients to ensure an accurate segmentation, by manually adjusting the parameters of the semi-automatic procedure.

The asymmetry level of each WM region was computed, taking as reference the hemisphere without the thalamic lesion $\left(1-\mathrm{Vol}_{\text {Lesioned }} / \mathrm{Vol}_{\text {Healthy }}\right)$. A control group of 14 age-matched epilepsy patients (6 females) (Suppl. Table 1) with no lesions reported in the MRI structural scans was processed similarly. For this group, the reference hemisphere was chosen to match the healthy hemisphere for each patient. A Shapiro-Wilk test was used to assess the normality of the data. The $z$-score of the asymmetry of each region was calculated and converted to a $p$-value using a two-sided z-test and an $\alpha$ of 0.05 .

Matlab was used to compute the correlation between the total volume of the atrophied WM regions and the loss of volume of the thalamic nuclei due to the lesions, using the corrcoef function. For each nucleus, the WM volume considered for this correlation was obtained by summing all the atrophied WM volumes in the lesioned hemisphere to which that nucleus projects. WM regions were considered atrophic, and thus included in this summation, when their asymmetry index exceeded the third quartile of the corresponding asymmetry distribution for the control group. To define the WM area corresponding to the Pulv area of projection, the following regions were included: banks of superior temporal sulcus; entorhinal; fusiform; inferior temporal; lingual; middle temporal; parahippocampal; superior temporal; supramarginal; temporal pole and transverse temporal. As for the MD, the corresponding regions were: lateral orbitofrontal; medial orbitofrontal; pars opercularis; pars orbitalis; pars triangularis; rostral middle frontal and frontal pole. These regions were selected based on (Sherman and Guillery 2013).

\section{Thalamic Connectivity Analysis}

In a subset of 4 patients, the MRI protocol included also a DWI acquisition, using the following parameters: echo planar imaging (EPI) with b-value of $1000 \mathrm{~s} / \mathrm{mm}^{2}$, voxel size of $2.3 \times 2.3 \times 2.3 \mathrm{~mm}^{3}, 52$ axial slices, TR/TE $=7200 / 97 \mathrm{~ms}$, 64 diffusion-weighting directions, plus 1 non-DWI image, for P1 and P2; EPI with b-value of $1000 \mathrm{~s} / \mathrm{mm}^{2}$, voxel size of $0.94 \times 0.94 \times 2.50 \mathrm{~mm}^{3}, 42$ axial slices, TR/ $\mathrm{TE}=8450 / 105.5 \mathrm{~ms}, 35$ diffusion-weighting directions, plus 1 non-DWI image, for P3 and P4. Non-DWI data were acquired at the beginning of the acquisition.

DWI data was corrected for geometrical distortions, using $e d d y$ from FMRIB's Diffusion Toolbox (FDT) (Andersson and Sotiropoulos 2016). Five cortical areas were defined using the Brainnetome atlas (Fan et al. 2016) in the MNI152 template: temporal, prefrontal, motor, and somatic, posterior parietal and occipital cortex according to (Behrens et al. 
2003). All masks were transformed into the DWI space by means of the Advanced Normalization Tools (ANTs) software (Avants et al. 2011) and FMRIB's Linear Image Registration Tool (FLIRT) (Jenkinson and Smith 2001). Probability distributions on 2 fiber directions were calculated at each voxel, using bedpostx from FDT (Behrens et al. 2003, 2007). From each voxel in the thalamus, 5000 samples were drawn to generate a connectivity distribution to each of the cortical masks. Each voxel was assigned to the cortical region to which it presented the highest connectivity (Behrens et al. 2003). The volume of each nucleus in each hemisphere was calculated, and the level of asymmetry between pairs was computed ( $\left.1-\mathrm{Vol}_{\text {Lesioned }} / \mathrm{Vol}_{\text {Healthy }}\right)$. The correlation between the total volume of the atrophied WM regions to which each thalamic nucleus projects and loss of volume of the thalamic nuclei was also evaluated considering the parcellation obtained according to the connectivity profile of the thalamus; to identify the two nuclei, we considered the Pulv and MD areas of projection to include the same regions as in Sect. 2.2.1. To validate the Pulv and the MD masks manually drawn on the structural images, the total probability of connection between these masks and each target region was computed, as a percentage of the total number of connections in the hemisphere and adjusted for the volume of the target region.

\section{EEG Data}

\section{Event-Related Potentials}

The functional status of the inferior occipital-temporal (O-T) brain areas was assessed using the N170 visual evoked potential, following a methodology previously published by our group (Lopes et al. 2011). Briefly, high-density EEG recordings were collected for all patients, with exception of P1, using a cap (EasyCap, Herrsching, Germany) with 78 electrodes, including all positions in the 10-10 system plus positions FT11, FT12, TP11, TP12. A collection of images of faces and words were presented twice on a computer screen, in a random sequence, with a stimulus duration of $250 \mathrm{~ms}$, and an interstimulus interval of $1 \mathrm{~s}$. A group of 10 aged-matched children (4 females) with Panayiotopoulos syndrome with normal cognitive development were also included in this study and used as a control group.

The EEG data were analysed with the software Source 2.0 (Compumedics-Neuroscan). First, the EEG data were filtered with high- and low-pass thresholds of respectively 1 and $70 \mathrm{~Hz}$. Electrocardiogram (ECG) and electrooculogram (EOG) artifacts were removed using a spatial filter. Using a standard realistic Finite Element Model (FEM) of the head, the model was fitted to the $\mathrm{N} 170$ scalp potential using two regional source dipoles placed at the centre position of each fusiform gyrus. The left/right ( $\mathrm{L} / \mathrm{R})$ hemisphere ratio was computed by taking the amplitude of each regional dipole at the peak of the Global Field Power (GFP) (Lopes et al. 2011).

\section{Epileptic Activity Analysis}

Long-term (24 h) EEG recordings were collected using a medical grade ambulatory EEG device (Trex HD, from Natus Inc), with a sampling rate of $200 \mathrm{~Hz}$ and a full set of 19 disc-shaped gold-plated electrodes. The electrodes were placed directly on the subject's head following the 10-20 system. For P2, subtemporal electrodes (F9/10 and P9/10) were also used. The spike index was calculated according to the methods described in Larsson et al. 2009 and Carvalho et al. 2020 and found to be consistently above $85 \%$.

The EEG data were filtered with high- and low-pass thresholds of respectively 0.5 and $70 \mathrm{~Hz}$ and with a $50 \mathrm{~Hz}$ notch filter. We performed automatic spike detection, using the Reveal algorithm as implemented in Persyst 13 (Scheuer et al. 2017), and also clustering. Spikes were grouped based on topology and morphology features - height, duration and tip angle - in a hierarchical fashion. The resulting dendrogram was opened until a leaf node (cluster) with a degree of dissimilarity equal or inferior to 4 was found. These final clusters were visually inspected: non-dipolar maps were detected and excluded by an experienced clinical neurophysiologist; the remaining clusters were subjected to a more detailed analysis, assessing the consistency of its spike components and the signal to noise ratio. Spikes within each cluster that survived the previous procedures were averaged, yielding epochs of $1 \mathrm{~s}$ each.

Localization of the spike activity of ESES was performed using a realistic boundary element model (BEM), obtained from the segmentation of the anatomical T1 image for each patient. Source analysis was performed with the standard low-resolution tomography (sLORETA) method (PascualMarqui 2002), in the cortical surface obtained with the BEM, and using standard electrical conductance values $(0.33,0.0042,0.33 \mathrm{~S} / \mathrm{m}$, respectively for scalp, skull, and brain (Geddes and Baker 1967)) and individual values for the scalp position of the cap EEG electrodes. For all individuals, scalp maps of each cluster, along with the respective source analysis, were recorded at the first two distinct and well-defined peaks of the GFP, since these are the points with best signal-to-noise ratio. MRI segmentation, BEM construction, and source analysis were done with the CURRY 6.0 software (Compumedics-Neuroscan).

\section{Results}

\section{WM Atrophy}

The WM analysis revealed several contiguous regions identified as being asymmetric, i.e., with different volume in the 
hemisphere of the thalamic lesion when compared to the healthy, contralateral side: the asymmetric regions for each patient are presented in Fig. 1 (Suppl. Table 2).

In the control group, the median level of asymmetry for each of the $34 \mathrm{WM}$ regions varied between $-30 \%$ and $+30 \%$ and also presented a wide dispersion of values (Fig. 2). This variability and also the non-normal distribution of values for some regions (darker boxplots in Fig. 2) led us to select as meaningfully asymmetric for the patients the WM regions with atrophy outside the third quartile of the control group. From a total of $76 \mathrm{WM}$ regions with meaningful atrophy in the seven patients, 43 (57\%) corresponded to areas of projection of the Pulv $(\mathrm{N}=27)$ or the MD $(\mathrm{N}=16)$ nuclei, with the remaining regions dispersed in areas of projection of other thalamic nuclei (Fig. 1). The predominance of asymmetries of Pulv cortical areas translates into the existence of temporal lobe WM atrophy in all the seven patients studied while only four patients had at least two MD cortical areas of atrophy over the frontal lobe.

\section{Thalamic Nuclei Volumetry}

In order to correlate the thalamic lesions with the abnormalities in WM, we quantified the volumes of the two major nuclei, the Pulv and MD nuclei (Fig. 3a), for which the main areas of cortical projection are well characterized (Sherman and Guillery 2013). The volumetric data for the Pulv revealed a volume loss ranging from $-2 \%$ to $69 \%$ (Suppl. Table 3). As for the MD, similar results were obtained, with a range of $-6 \%$ to $69 \%$.
Fig. 1 From left to right: lateral, medial, inferior and superior views of the inflated lesioned hemisphere of each subject.

The gray regions correspond to the 34 regions of the DesikanKilliany atlas identified with Freesurfer. Colors indicate the regions for which the WM was found to be meaningfully asymmetric in each patient in the Pulv major cortical projection areas (Red); MD major cortical projection areas (Blue); projection areas of other thalamic nuclei (Green). Magenta circles: sLORETA solution for each cluster at the first well-defined peak of the GFP; Yellow circles: sLORETA solution for each cluster at the second well-defined peak of the GFP; Diamond shapes: sLORETA solution for the main cluster. P3, $\mathrm{P} 6$, and $\mathrm{P} 7$ have left thalamic lesions, while the others have right thalamic lesions

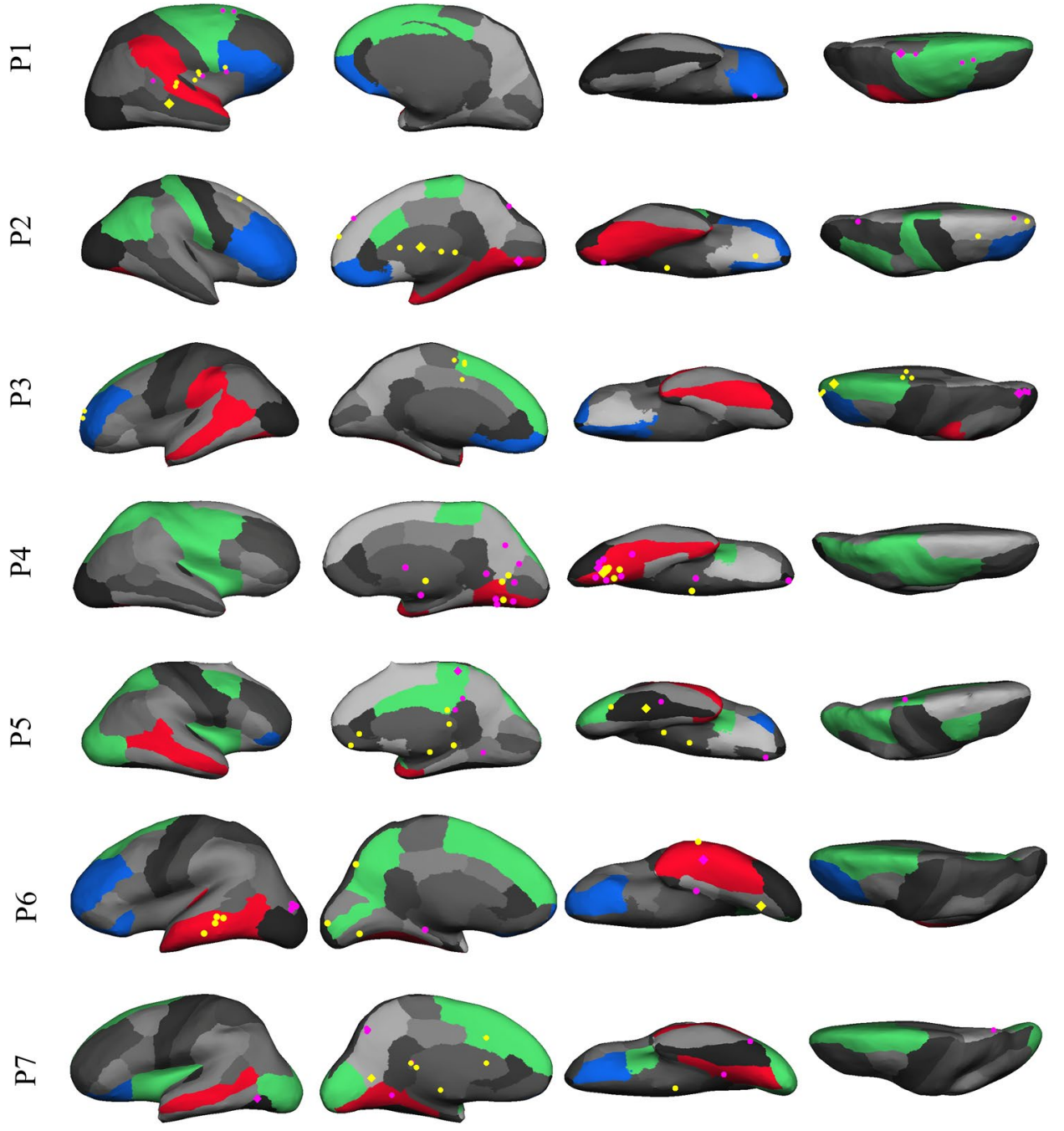

Pulv $\square$ MD $\square \begin{aligned} & \text { Other } \\ & \text { nuclei }\end{aligned}$ Dipole at peak $1 \bigcirc$ Dipole at peak 2

Dipole of main cluster at peak $1 \bigcirc$ Dipole of main cluster at peak 2 
Fig. 2 Distribution of WM asymmetries for controls $(\mathrm{N}=14)$ for each of the WM regions identified-boxplots. Patient data are represented with symbols. The top graph considers the left as the healthy hemisphere, therefore the points represented are the right lesioned patients. In the bottom graph, the healthy hemisphere is assumed to be the right one, thus the represented data is relative to the left lesioned patients. The anatomical areas for which the distribution for the controls was not normal, according to the Shapiro-Wilk test, are depicted with darker boxplots; the areas for which the asymmetry was found to be significant (p-value <0.05) are identified with the filled markers
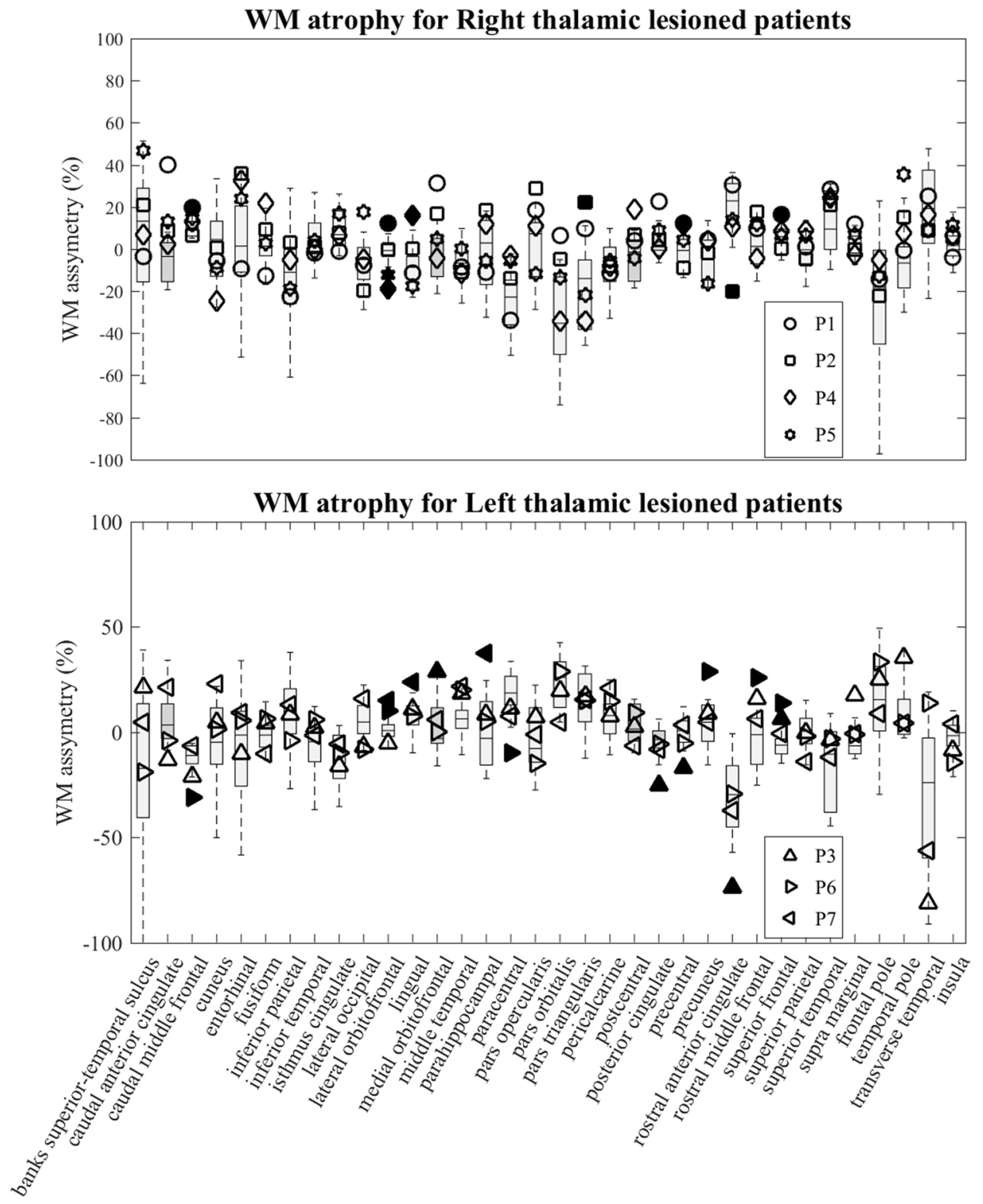

By plotting the WM volume of the atrophic regions in the lesioned hemisphere that lay in the area of connection of the Pulv (Fig. 3b-left) and MD (Fig. 3b-right) as a function of the atrophy of each nucleus, a linear relationship between them can be seen, suggesting that a larger volume loss of a thalamic nucleus translates into a larger atrophy of the WM connecting to its cortical projecting areas. A significant positive correlation was found between the total volume of the WM atrophied regions and nuclei atrophy for both the Pulv $(\mathrm{r}=0.83, \mathrm{p}<0.020)$ and MD $(\mathrm{r}=0.83, \mathrm{p}<0.020)$. The broad confidence intervals $(0.211$ to 0.975 and 0.208 to 0.974 for Pulv and MD, respectively) are likely to be due to the small dimension of our sample.

\section{Event-Related Potentials}

The N170 potential inter-hemispheric ratios for both faces and words obtained for the group of controls and patients are presented in Fig. 4 (and Suppl. Table 4). In the control group, the N170 ratio showed slightly different values around 1, for the two types of stimuli, in accordance with results of previous studies with similar protocols (DaviesThompson et al. 2016).

Of the two patients with no evidence of inferior O-T WM atrophy, one was not studied (P1) and the other (P5) had normal N170 ratios, both for faces and words. Of the five patients with inferior O-T WM atrophy, four (P2, P3, P4, and 
(a)
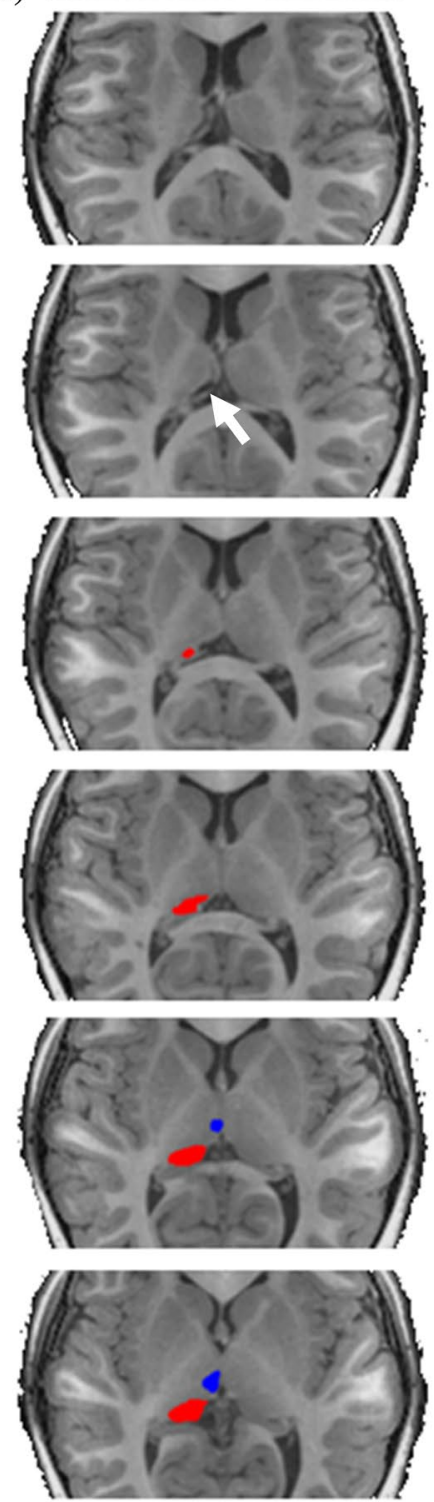

$\mathrm{R}$ (b)

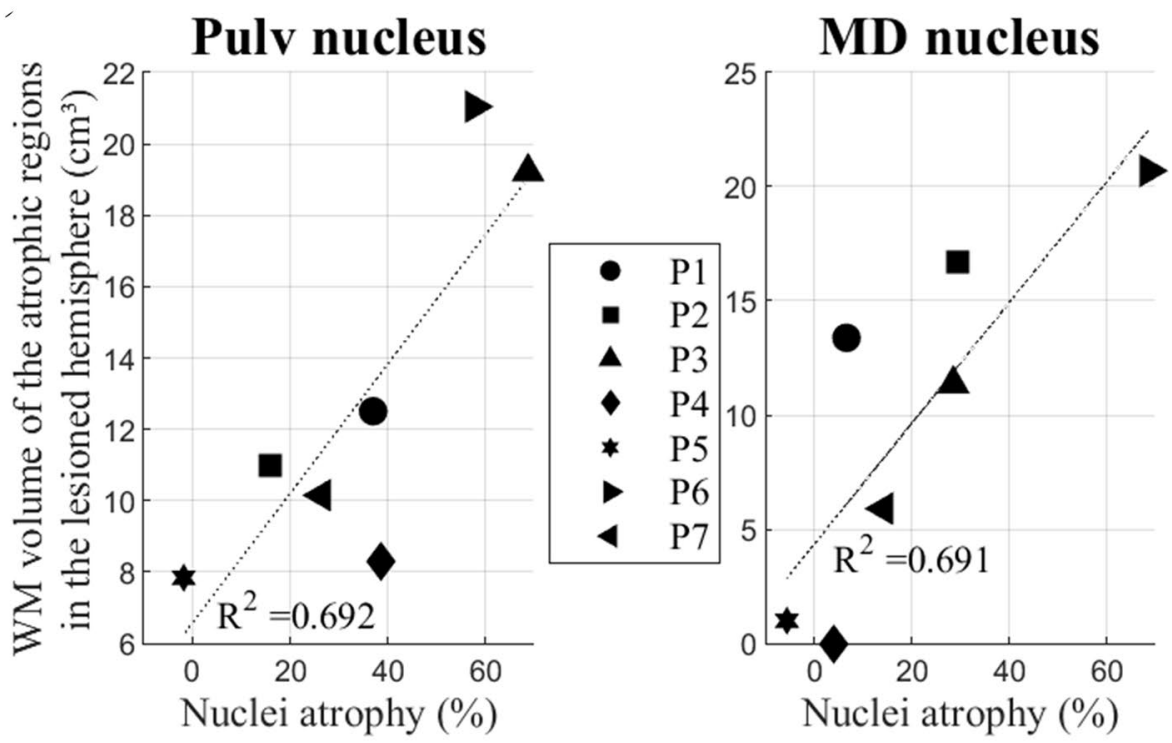

(c)

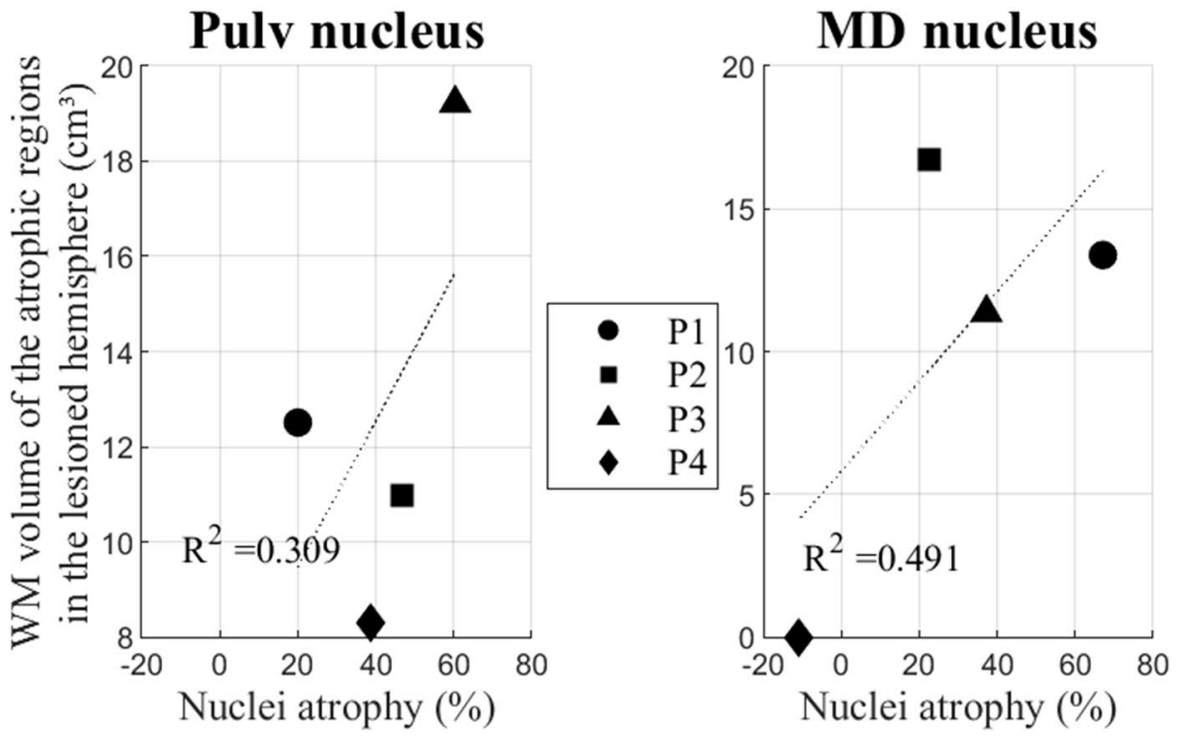

Fig. 3 a Axial slices of structural MRI from P5. The arrow indicates the thalamic lesion, the Pulv nucleus is illustrated in red and the MD in blue. $\mathbf{b}$ WM volume of the atrophic regions in the lesioned hemisphere as a function of the Pulv (left) and MD (right) nuclei atrophy. It can be seen that the volume of the atrophic regions increases with the thalamic nuclei atrophy for both the Pulv and MD nuclei. c

P7) had at least one pathological ratio, while the remaining one (P6) had a face N170 ratio at the extreme of normal control range. Two cases with WM atrophy in the right hemisphere ( $\mathrm{P} 2$ and $\mathrm{P} 4)$ presented exclusively abnormal face N170 ratios, while two of the three cases with atrophy in the left hemisphere (P3 and P7) had predominantly word N170 abnormal ratios (Fig. 4 and Suppl. Table 4).
Correlation between the WM volume of the atrophic regions and the thalamic nuclei obtained with the DWI connectivity parcellation for patients $\mathrm{P} 1, \mathrm{P} 2, \mathrm{P} 3$, and $\mathrm{P} 4$. A positive correlation supports the association of WM atrophy and thalamo-cortical disconnection both for Pulv and MD nuclei

These results are consistent with the well-known dominance of the right hemisphere for faces and the left hemisphere for word processing and support the existence in our patients of cortical dysfunction associated with inferior O-T WM atrophy. 


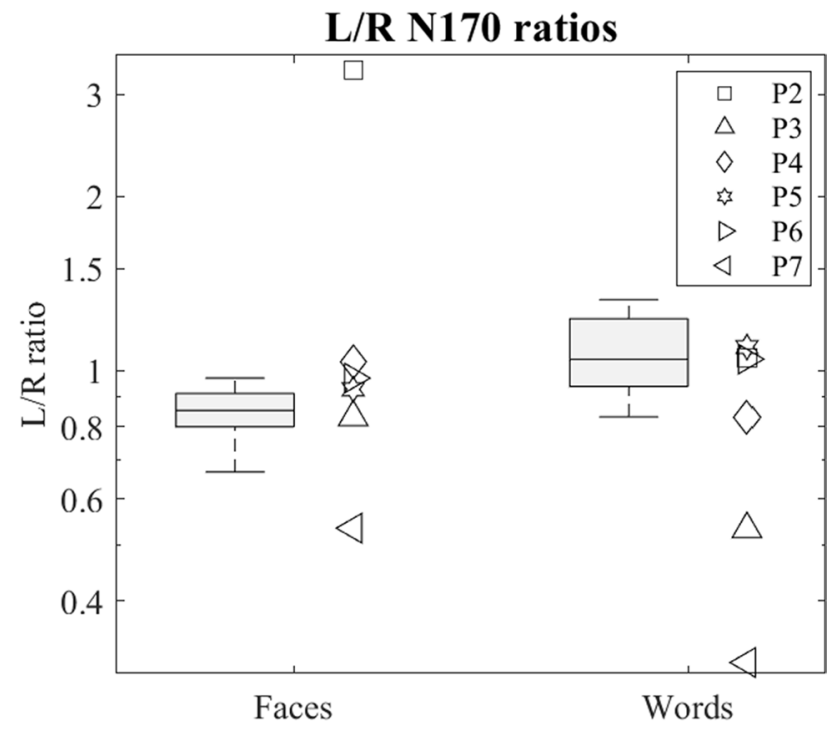

Fig. 4 Ratio between left and right hemisphere N170 amplitudes for the presentation of faces (left) and words (right). Boxplots represent the distribution in the control group $(\mathrm{N}=10)$, and symbols indicate the individual patients. The control range for faces showed lower amplitude ratios when compared to the one for words. P2, P4, and P7 have asymmetrical N170 potentials for faces, outside the range of the control group. P3 and P7 have abnormal N170 ratios, outside the range of the control group, for words. The triangles show the left lesioned patients

\section{Epileptic Activity Distribution}

The spike clustering algorithm identified 9, 8, 16, 14, 10, 8 and 8 clusters, for subjects $\mathrm{P} 1$ to $\mathrm{P} 7$, respectively, which finally lead to $8,7,16,14,9,8$ and 6 after visual inspection to remove non-dipolar clusters.

The main cluster, which had substantially more spikes compared to the others, had 29,421, 42,137, 16,848, 19,119, $31,932,39,820$ and 39,571 spikes for patients 1 to 7 , respectively. It is worth noting that for P1, P5 and P7 the corresponding EEG data had to be cropped, discarding 2 to 3 sleep cycles, given that a large number of spikes had been detected, resulting in datasets too large to be processed by the 6.0 GB RAM machine that was used to run the spike clustering algorithm.

The average potential topographic maps for representative spikes produced dipolar fields with stable spatial configurations around the peaks, suggesting stable underlying intracranial generators and with potential phase reversal lateralized to the hemisphere of the thalamic lesion. Figure 5 illustrates these dipolar fields around the two peaks of the GFP for the main cluster of each patient, as well as the respective source maps obtained with sLORETA. The results for the remaining clusters can be found in Suppl. Figure 1.

The visual analysis of the orientation of the potential fields over the scalp and the corresponding sLORETA solution for the main cluster revealed distinct source patterns for the different patients: P1 had two sources, localized in the temporal-frontal and superior frontal cortex of the right hemisphere; for $\mathrm{P} 2, \mathrm{P} 4$ and $\mathrm{P} 7$, source solutions were obtained in the inferior O-T cortex, that remained localized in $\mathrm{P} 4$ and $\mathrm{P} 7$, but associated with a deep middle frontal source in P2; for P3 a left occipital source associated with a left frontal one, both near the middle line; P5 associated parietal and a deep occipital sources near the middle line; P6 presented inferior temporal and O-T sources.

As for the remaining clusters, the intracranial generators that were at the origin of the epileptic activity for each of those, for two distinct peaks, are overlaid in the inflated brain of each subject (Fig. 1). For P1, P3, P4, and P6, the source localizations of the secondary clusters were distributed around the main one, while for P2, P5 and P7 they were more widely scattered.

\section{Thalamic Connectivity Analysis}

Examining the connectivity of the manually drawn Pulv and MD nuclei allows us both to determine differences between the healthy and lesioned nuclei and also to validate those masks using the connectivity results. The manually drawn Pulv and MD nuclei approximately correspond to the temporal and prefrontal nuclei identified with tractography, respectively (Fig. 6). The positive correlation between the volume of the atrophied WM regions to which either the Pulv or MD nuclei project and the loss of volume of each of these nuclei (Fig. 3c) is similar to the one found in Fig. 3b. Here, no significant correlation was found for any of the nuclei: Pulv $(\mathrm{r}=0.56, \mathrm{p}<0.444)$ and MD $(\mathrm{r}=0.70, \mathrm{p}<0.299)$. Moreover, the confidence intervals ( -0.870 to 0.989 and -0.797 to 0.993 for Pulv and MD, respectively) are even wider than those shown in Fig. 3b, since the sample size is smaller in this case. Even though the correlation between the volume of WM atrophied areas and the loss of volume of the thalamic nuclei due to the lesions is more evident in the results shown in Fig. 3b than Fig. 3c, both observations support the interpretation that the loss of thalamic volume results in decreased thalamo-cortical connectivity and WM atrophy in relation with the respective cortical projection areas.

\section{Discussion}

The present study puts in evidence widespread regional WM atrophy in the hemispheres with thalamic lesions, which is associated with cortical dysfunction as well as with the spatial localization of the epileptic activity underlying the ESES. 
Peak 1

$\bar{a}$
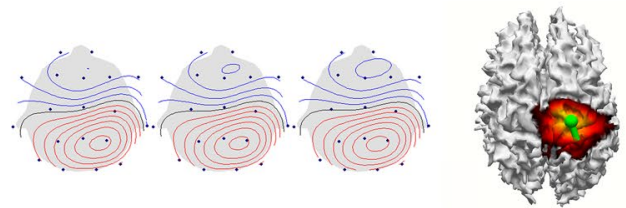

ล
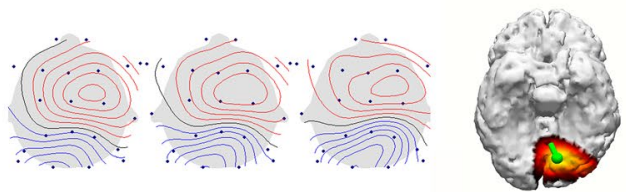

$\Omega$
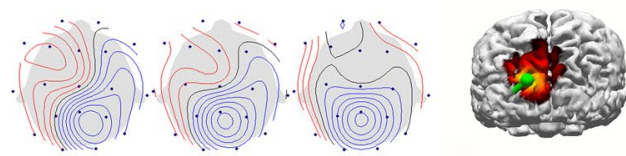

I
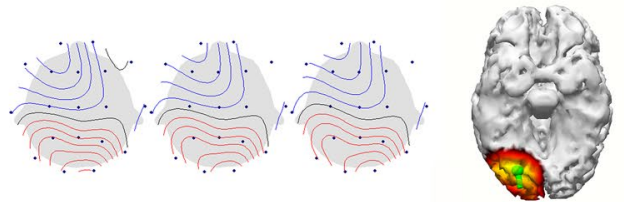

$\approx$
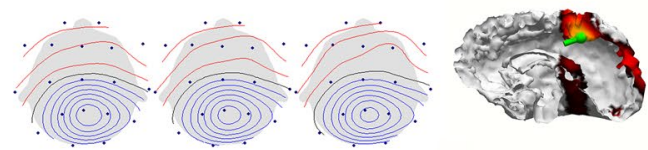

$\stackrel{\circ}{2}$
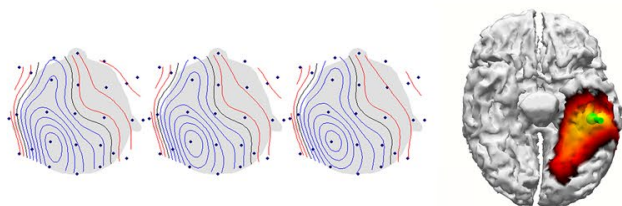

$\hat{2}$

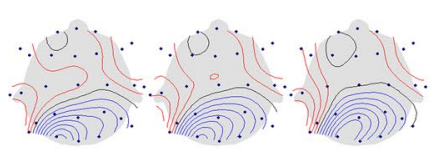

Max

L

$\mathrm{R}$
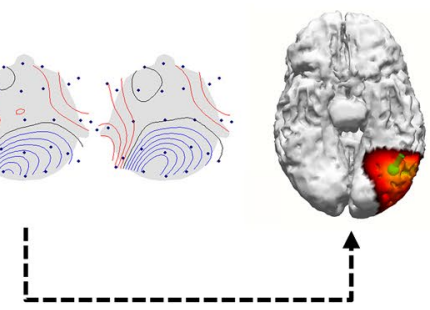

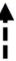

Peak 2
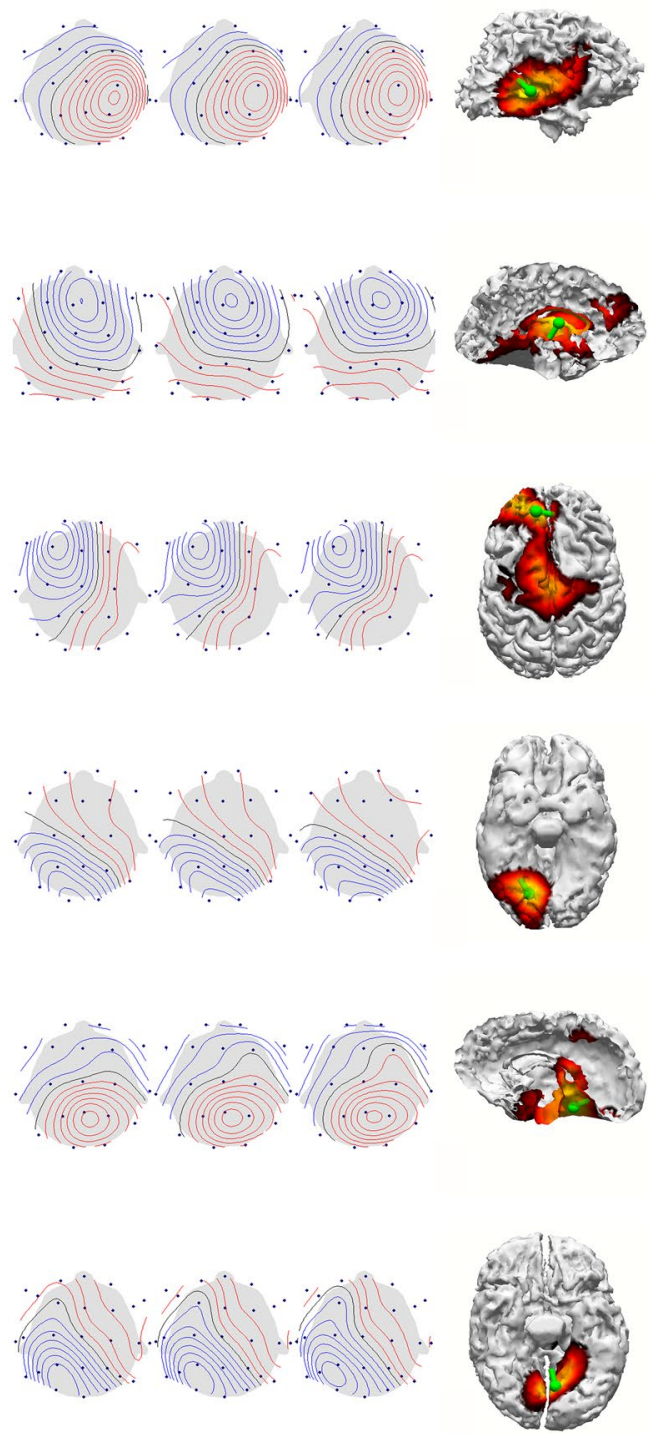

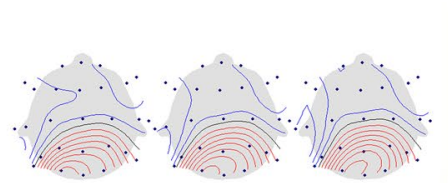

L $\quad$ R

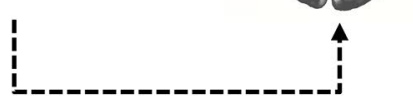

$75 \%$ Max

Fig. 5 Topography maps (left) for three time points and respective source maps obtained for the middle source map with sLORETA (right), for the main cluster of spikes identified in each subject. The two columns represent maps obtained at the first and second peaks of the GFP. Source maps were threshold at $75 \%$ of their maximum scores 


\section{Pulv nucleus}
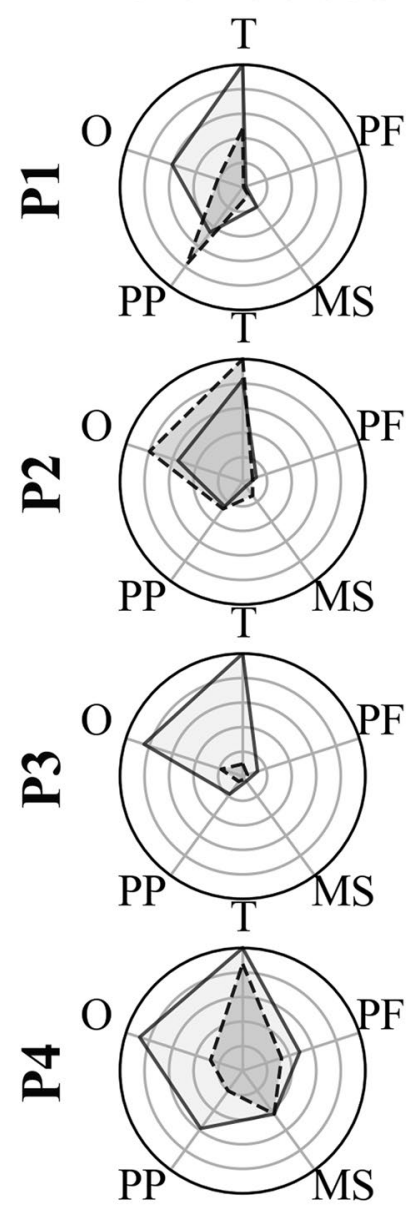

MD nucleus
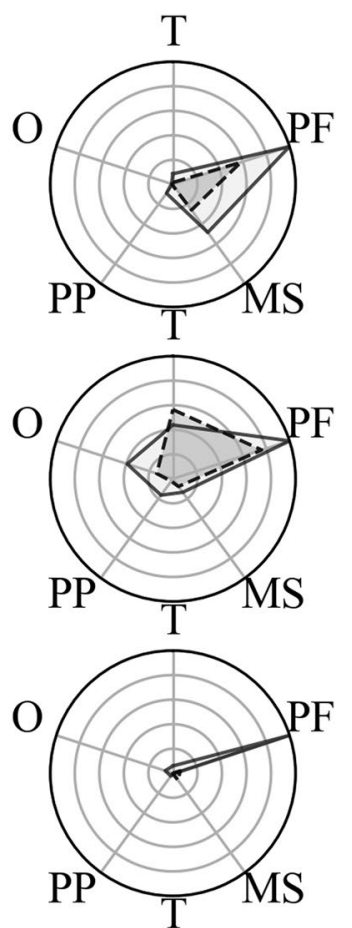

$\mathrm{O}$

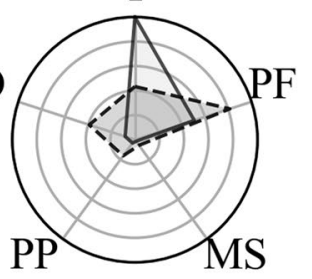

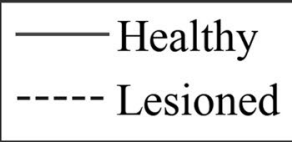

Fig. 6 Total probability of connection of WM tracts between the Pulv and MD manually drawn masks and each target region, as a percentage of total connections in the hemisphere and adjusted for the volume of the target region. The limits of the plots were adjusted for each case, to allow a better visualization of the distribution, therefore any comparison of values between the plots would be inaccurate. $T$ temporal, $P F$ prefrontal, $M S$ motor and somatic, $P P$ posterior parietal, $O$ occipital cortices. The lesioned nuclei exhibited less connectivity with the respective cortical areas when compared to the corresponding healthy side. The Pulv nuclei showed a wider distribution of connectivity, which included mainly the temporal and the occipital cortex

\section{WM Atrophy Correlates with Thalamic Nuclei Atrophy}

The overall pattern of thalamic lesions in the patients studied has predominantly a periventricular distribution (Fig. 3a), (Fig. 2 of Leal et al. 2018), which is similar to one of the

other published cases with the same etiology (Guzzetta et al. 2005; Kelemen et al. 2006; Losito et al. 2015; Monteiro et al. 2001), and affects mainly nuclei of the dorsal thalamus, namely the Pulv and MD nuclei. Due to the destructive character of the thalamic haemorrhage, one expects that fibers projecting from that region to the cortex and vice-versa would cease to exist or get rearranged, producing volumetric changes in the contiguous WM region of the affected hemisphere (Zuo et al. 2017). The positive linear correlation found between the volumetric loss of both the Pulv and MD nuclei and the volume of the atrophied WM regions in relation with the corresponding cortical projection areas (Fig. 3b) supports this hypothesis, by demonstrating that larger thalamic lesions are associated with a higher number of WM regions with marked atrophy, and therefore a larger total volume of atrophied WM. A similar correlation was found using probabilistic tractography (Fig. 3c) further suggesting that the decrease in thalamo-cortical connectivity drives WM atrophy.

Overall, our results support the interpretation that the thalamic lesions suffered by our patients, early in development, produced a decrease in the thalamo-cortical structural connectivity, which led to regional WM atrophy.

\section{WM Anatomical Atrophy Produces Cortical Dysfunction}

The inferior O-T cortex is an area of projection of the Pulv (Sherman and Guillery 2013) and it shows WM corresponding atrophy in most of our patients, in line with the lesions of this nucleus. This cortical region is well-known to participate in high-level processing of visual information, and in particular to generate the N170 potential evoked by complex stimuli such as faces and words (Cohen et al. 2002; DaviesThompson et al. 2016) The results for the N170 protocol demonstrated abnormalities that correlated not only with the inferior O-T-related WM atrophy but also were in line with the hemispheric laterality. In fact, patients with right hemisphere inferior O-T-related WM atrophy (P2 and P4) produced abnormal N170 for faces, while two of the three patients with such atrophy in the left hemisphere (P3 and P7) produced abnormal N170 predominantly for words (Fig. 4).

These abnormalities of the N170 are similar to the ones previously demonstrated in patients with inferior temporal lobe lesions with symptomatic focal epilepsies (Lopes et al. 2011) and support the interpretation that the WM atrophy in inferior O-T regions of our patients produces an impairment of the functional role of these cortical areas in high-level visual processing. The alternative hypothesis that the N170 abnormalities might be due to the ongoing epileptic activity seems to us less likely because such potentials have been recorded with patients fully awake when little or no spike activity was present. 
The comparison of N170 abnormalities with the distribution of spike sources reveals that in the four patients with clearly pathological ratios ( $\mathrm{P} 2, \mathrm{P} 3, \mathrm{P} 4$, and $\mathrm{P} 7)$ there was a main cluster of spikes over the occipital lobe of the affected hemisphere, suggesting that the dysfunction detected while awake largely overlaps with areas of significant spiking during sleep. Despite this, in the two remaining patients assessed, the main spike cluster is located in the fusiform areas without a resulting abnormal N170 ratio. Comparatively to this $4 / 6$ probability that an N170 abnormal ratio is associated with the main spike cluster over the occipital lobe, the occurrence of WM atrophy over this lobe was associated with a $5 / 6$ probability that the main spike cluster was also located there (P2, P3, P4, P5, P6, and P7). These data suggest that WM atrophy has a stronger predictability power for the localization of spike activity than the N170 abnormalities.

The neurocognitive evaluation of the patients was driven by the tools available to the attending clinician; this was very sparse and not uniform in time, which did not allow the construction of a reliable disability scale that could be correlated with the WM atrophy. It is nevertheless apparent that the patient with the highest overall WM atrophy (P6) is one of the most severely disabled (Table 1), while patient P4, who showed the least WM atrophy, exhibited no apparent disability. The latter observation must be tempered by the existence of only an early formal assessment (at age 2 years) and a limited follow-up.

\section{WM Atrophy Correlates with Epileptic Activity Distribution}

The spike cluster analysis put in evidence that the main cluster contained an overwhelming number of spikes as compared with secondary clusters. This is in line with the clinical observation that despite the fact that patients with ESES often present with multifocal spikes, not all spikes activate in the same way at sleep onset (Gibbs et al. 2019).

Two patterns were apparent for the main clusters sources: a focal pattern where the sources associated with the first and second peaks of the GFP were localized in the same cortical region $(\mathrm{P} 4, \mathrm{P} 6$, and $\mathrm{P} 7)$; and a multifocal pattern where the two sources were widely separated, suggesting the existence of more than one epileptogenic area (P1, P2, P3, and P5). In the patients with the focal pattern, sources were located in the inferior or lateral O-T region, where several areas of WM atrophy were also localized (Fig. 1). The multifocal group was more heterogeneous: $\mathrm{P} 1$ presented sources in the perisylvian and superior frontal areas, both overlapping areas of WM atrophy; P2 sources localized in the inferior O-T and deep middle line frontal areas, both overlapping regions of WM atrophy; P3 sources were localized in frontal and occipital regions, but only the first overlapped WM areas of atrophy; P5 sources in the superior parietal and medial O-T regions also demonstrated overlap with areas of WM atrophy. Overall, we found a good spatial correlation between WM atrophy regions and spike sources in five patients and a partial one in the other two.

Other etiologies of ESES involving the thalamus have been described, namely in polymicrogyria, where a good correlation between the syndrome and volumetric reductions of the thalamus was shown (Bartolini et al. 2016). These authors describe, in a sample of 27 retrospectively studied, patients with cortical malformation, a high predictability from thalamic volume reductions for evolution to ESES. The extensive and early onset associated to cortical malformations in such patients make the identification of a critical mechanism for ESES more difficult than in our sample of patients with lesions limited to the thalamus and without cortical malformations. In fact, the high probability of evolution to ESES in patients with thalamic neonatal lesions (van den Munckhof et al. 2020) suggests that such lesions, developing at a time when the brain cortical-thalamic anatomy is already well established (neonatal period), are able to trigger all features of this encephalopathy without the need for associated cortical malformations.

\section{Role of WM Regional Atrophy in the ESES}

If we assume that the pathophysiological mechanism leading from early thalamic lesions to ESES is similar to the one of other etiologies, then a macroscopic thalamic lesion is not a requirement as it is only found in a small minority of cases (Sánchez Fernández et al. 2012a). Alternatively, if the critical factor is a WM lesion leading to cortical disconnection, then other ESES etiologies can be included, as regional lesions of the WM are present in a large subset of reported etiologies (Sánchez Fernández et al. 2013, 2012b; Guzzetta et al. 2005; Losito et al. 2015) including: vascular lesions; periventricular leukomalacia; delayed myelination; hydrocephalus; hemispheric atrophy; agenesis of the corpus callosum; non-specific lesions on T2-weighted MRI.

Several lines of evidence point to the cortex as the origin of spike activity in ESES (Siniatchkin et al. 2010), but the striking results of surgical hemispherectomy, a procedure that disconnects the cortex from the brainstem and thalamus, with a fast disappearance of this epileptic pattern (reviewed in Jansen et al. 2019), suggests that a subcortical connection is ESES for its expression. If we take into consideration that an early thalamic lesion can by itself lead to ESES (Kersbergen et al. 2013), then the likely subcortical structure critical for the ESES cortical expression is the thalamus. A more detailed anatomical analysis by Leal et al. 2018 further suggests that most, if not all, thalamic lesion cases are associated with the dorsal thalamus while the ventral structures are not visibly involved. 
Overall, the combination of results from the present study and data from the literature suggests that the critical factor favoring the expression of ESES type of spike activity in a particular cortical area is its disconnection from the dorsal thalamus while preserving the connectivity to the ventral thalamus. WM abnormalities potentially leading to this outcome can be found in a much wider range of brain lesions historically associated with ESES than the rare early thalamic lesion cases.

\section{Limitations}

In this study, the healthy hemisphere, which was taken as reference, was considered to be the one contralateral to the thalamic lesion. Nonetheless, this is not always completely accurate: P1, for example, presented a great enlargement of the ventricles in both hemispheres, making this referencing a limitation.

Manually drawing the thalamic nuclei masks is hard due to the limited contrast of the MRI structural scans and the results can be affected by the experience of the user. For this reason, several studies have performed automatic parcellation of the human thalamus instead, using DWI and probabilistic tractography to differentiate nuclei based on their connectivity profiles (Behrens et al. 2003; JohansenBerg et al. 2005; Broser et al. 2011; O'Muircheartaigh et al. 2011). Nevertheless, the tractography method per se is not able to define the fiber polarity, which could be advantageous to understand exactly which fibers are involved in the dysconnectivity. Also, this methodology is mostly sensitive to major pathways; consequently, the hemispheric asymmetries seen are limited to the dominant pathways in each nucleus.

Because of the retrospective exploratory character of the present study, the data used in this study was not collected at the same time, nor using the same acquisition protocols; and so the results may be affected by differences in the acquisition equipment and imaging protocols, which can justify some of the differences seen between subjects.

Finally, the reduced sample size constitutes a significant limitation to this study, which did not allow us to reach strong statistical correlations. Our exploratory conclusions will benefit greatly from a larger effort of recruitment to either confirm or disprove them. We also acknowledge that having a control group of subjects with thalamic lesions and no epilepsy would have been beneficial for the study. However, isolating such a group is extremely difficult due to the fact that early thalamic lesions are very rare conditions, and a control group of such cases with no ESES is even rarer, as the majority of such patients evolve to this syndrome (van den Munckhof et al. 2020). The evaluation of the neurocognitive-behavioral disability was also limited by the use of different tools and temporally sparse assessments.

\section{Conclusion}

In our study, we demonstrate a good correlation between early neonatal thalamic lesions in 7 patients with ESES and associated regional WM atrophy. Using visual evoked potentials, we found abnormal responses of the atrophic regions supporting a dysfunctional effect, and source analysis showed a good overlap with the generators of spike activity.

The association between regional WM atrophy and sources of spike activity with the features seen in ESES is an important one as these structural abnormalities can be found in other etiologies of this syndrome besides neonatal thalamic hemorrhages (reviewed in Sánchez Fernández et al. 2012a). If the critical factor leading to the onset of ESES proves to be the thalamo-cortical disconnection, then the model proposed to account for the origin of this syndrome in cases of early thalamic lesions (Leal et al. 2018) could be applied to a more widespread group of developmental lesions in early childhood. The validation of a common pathological mechanism at work at the multiple etiologies described for the ESES would be a major step forward in our understanding of this mysterious syndrome.

Acknowledgements This work was supported by the Portuguese Science Foundation (FCT) through Project UID/EEA/50009/2019.

\section{Compliance with Ethical Standards}

Competing interests The authors declare no competing interests.

\section{References}

Andersson JLR, Sotiropoulos SN (2016) An integrated approach to correction for off-resonance effects and subject movement in diffusion MR imaging. Neuroimage 125:1063-1078. https://doi. org/10.1016/j.neuroimage.2015.10.019

Avants BB, Tustison NJ, Song G et al (2011) A reproducible evaluation of ANTs similarity metric performance in brain image registration. Neuroimage 54:2033-2044. https://doi.org/10.1016/j. neuroimage.2010.09.025

Bartolini E, Falchi M, Zellini F et al (2016) The syndrome of polymicrogyria, thalamic hypoplasia, and epilepsy with CSWS. Neurology 86:1250-1259. https://doi.org/10.1212/WNL.00000 00000002526

Behrens TEJ, Berg HJ, Jbabdi S et al (2007) Probabilistic diffusion tractography with multiple fibre orientations: What can we gain? Neuroimage 34:144-155. https://doi.org/10.1016/j.neuro image.2006.09.018

Behrens TEJ, Woolrich MW, Smith SM et al (2003) Non-invasive mapping of connection between human thalamus and cortex using diffusion imaging. Nat Neurosci 6:750-757. https://doi. org/10.1038/nn1075

Broser P, Vargha-khadem F, Clark CA (2011) Robust subdivision of the thalamus in children based on probability distribution 
functions calculated from probabilistic tractography. Neuroimage 57:403-415. https://doi.org/10.1016/j.neuroimage .2011 .04 .054

Byne W, Buchsbaum MS, Kemether E et al (2001) Magnetic Resonance Imaging of the Thalamic Mediodorsal Nucleus and Pulvinar in Schizophrenia and Schizotypal Personality Disorder. Arch Gen Psychiatry 58:133-140. https://doi.org/10.1001/archpsyc.58.2.133

Carvalho D, Mendes T, Dias AI, Leal A (2020) Interictal spike quantification in continuous spike-wave of sleep (CSWS): Clinical usefulness of a wearable EEG device. Epilepsy Behav 104:106902. https://doi.org/10.1016/j.yebeh.2020.106902

Cohen L, Lehéricy S, Chochon F et al (2002) Language-specific tuning of visual cortex? Functional properties of the Visual Word Form Area. Brain 125:1054-1069. https://doi.org/10.1093/brain/awf094

Davies-Thompson J, Johnston S, Tashakkor Y et al (2016) The relationship between visual word and face processing lateralization in the fusiform gyri: a cross-sectional study. Brain Res 1644:88-97. https://doi.org/10.1016/j.brainres.2016.05.009

Desikan RS, Ségonne F, Fischl B et al (2006) An automated labeling system for subdividing the human cerebral cortex on MRI scans into gyral based regions of interest. Neuroimage 31:968-980. https://doi.org/10.1016/j.neuroimage.2006.01.021

Dorris L, O'Regan M, Wilson M, Zuberi SM (2019) Progressive intellectual impairment in children with Encephalopathy related to Status Epilepticus during slow Sleep. Epileptic Disord 21(S1):S88S96. https://doi.org/10.1684/epd.2019.1063

Fan L, Li H, Zhuo J et al (2016) The human brainnetome atlas: a new brain atlas based on connectional architecture. Cereb Cortex 26:3508-3526. https://doi.org/10.1093/cercor/bhw157

Fernandez-Miranda JC, Pathak S, Engh J et al (2012) High-definition fiber tractography of the human brain: neuroanatomical validation and neurosurgical applications. Neurosurgery 71:430-453. https ://doi.org/10.1227/NEU.0b013e3182592faa

Geddes LA, Baker LE (1967) The specific resistance of biological material - a compendium of data for the biomedical engineer and physiologist. Med Biol Eng 5:271-293. https://doi.org/10.1007/ BF02474537

Gibbs SA, Nobili L, Halász P (2019) Interictal epileptiform discharges in sleep and the role of the thalamus in Encephalopathy related to Status Epilepticus during slow Sleep. Epileptic Disord 21(S1):S54-S61. https://doi.org/10.1684/epd.2019.1058

Guzzetta F, Battaglia D, Veredice C et al (2005) Early thalamic injury associated with epilepsy and continuous spike-wave during slow sleep. Epilepsia 46:889-900. https://doi.org/10.111 1/j.1528-1167.2005.64504.x

Incorpora G, Pavone P, Smilari PG et al (1999) Late primary unilateral thalamic hemorrhage in infancy: report of two cases. Neuropediatrics 30(5):264-267. https://doi.org/10.1055/s-2007-973501

Jansen FE, Nikanorova M, Peltola M (2019) Current treatment options for Encephalopathy related to Status Epilepticus during slow Sleep. Epileptic Disord 21(S1):S76-S81. https://doi.org/10.1684/ epd.2019.1061

Jenkinson M, Smith S (2001) A global optimisation method for robust affine registration of brain images. Med Image Anal 5:143-156. https://doi.org/10.1016/S1361-8415(01)00036-6

Johansen-Berg H, Behrens TEJ, Sillery E et al (2005) Functional-anatomical validation and individual variation of diffusion tractography-based segmentation of the human thalamus. Cereb Cortex 15:31-39. https://doi.org/10.1093/cercor/bhh105

Kelemen A, Barsi P, Gyorsok Z et al (2006) Thalamic lesion and epilepsy with generalized seizures, ESES and spike-wave paroxysms-Report of three cases. Seizure 15:454-458. https://doi. org/10.1016/j.seizure.2006.05.006

Kersbergen KJ, de Vries LS, Leijten FSS et al (2013) Neonatal thalamic hemorrhage is strongly associated with electrical status epilepticus in slow wave sleep. Epilepsia 54:733-740. https:// doi.org/10.1111/epi.12131

Larsson PG, Wilson J, Eeg-Olofsson O (2009) A new method for quantification and assessment of epileptiform activity in EEG with special reference to focal nocturnal epileptiform activity. Brain Topogr 22:52-59. https://doi.org/10.1007/s10548-008-0072-3

Leal A, Calado E, Vieira JP et al (2018) Anatomical and physiological basis of continuous spike-wave of sleep syndrome after early thalamic lesions. Epilepsy Behav 78:243-255. https://doi. org/10.1016/j.yebeh.2017.08.027

Lopes R, Cabral P, Canas N et al (2011) N170 asymmetry as an index of inferior occipital dysfunction in patients with symptomatic occipital lobe epilepsy. Clin Neurophysiol 122:9-15. https://doi. org/10.1016/j.clinph.2010.05.023

Lopes R, Simões MR, Leal AJR (2014) Neuropsychological abnormalities in children with the Panayiotopoulos syndrome point to parietal lobe dysfunction. Epilepsy Behav 31:50-55. https://doi. org/10.1016/j.yebeh.2013.11.013

Losito E, Battaglia D, Chieffo D et al (2015) Sleep-potentiated epileptiform activity in early thalamic injuries: study in a large series (60 cases). Epilepsy Res 109:90-99. https://doi.org/10.1016/j.eplep syres.2014.10.015

Monteiro JP, Roulet-Perez E, Davidoff V, Deonna T (2001) Primary neonatal thalamic haemorrhage and epilepsy with continuous spike-wave during sleep: a longitudinal follow-up of a possible significant relation. Eur J Paediatr Neurol 5:41-47. https://doi. org/10.1053/ejpn.2001.0403

O'Muircheartaigh J, Vollmar C, Traynor C et al (2011) Clustering probabilistic tractograms using independent component analysis applied to the thalamus. Neuroimage 54:2020-2032. https://doi. org/10.1016/j.neuroimage.2010.09.054

Pascual-Marqui RD (2002) Standardized low resolution brain electromagnetic tomography (sLORETA): technical details. Methods Find Exp Clin Pharmacol 24(Suppl D):5-12

Power BD, Wilkes FA, Hunter-Dickson M et al (2015) Validation of a protocol for manual segmentation of the thalamus on magnetic resonance imaging scans. Psychiatry Res Neuroimaging 232:98105. https://doi.org/10.1016/j.pscychresns.2015.02.001

Sánchez Fernández I, Takeoka M, Tas E et al (2012a) Early thalamic lesions in patients with sleep-potentiated epileptiform activity. Neurology 78:1721-1727. https://doi.org/10.1212/WNL.0b013 e3182582ff8

Sánchez Fernández I, Loddenkemper T, Peters JM, Kothare SV (2012b) Electrical status epilepticus in sleep: clinical presentation and pathophysiology. Pediatr Neurol 47:390-410. https://doi. org/10.1016/j.pediatrneurol.2012.06.016

Sánchez Fernández I, Chapman KE, Peters JM et al (2013) Continuous spikes and waves during sleep : electroclinical presentation and suggestions for management. Epilepsy Res Treat 2013:1-12. https ://doi.org/10.1155/2013/583531

Scheuer ML, Bagic A, Wilson SB (2017) Spike detection: Interreader agreement and a statistical Turing test on a large data set. Clin Neurophysiol 128:243-250. https://doi.org/10.1016/j.clinp h.2016.11.005

Schiffler P, Tenberge J-G, Wiendl H, Meuth SG (2017) Cortex parcellation associated whole white matter parcellation in individual subjects. Front Hum Neurosci 11:1-10. https://doi.org/10.3389/ fnhum.2017.00352

Sherman SM, Guillery RW (2013) Functional connections of cortical areas-a new view from the thalamus. MIT Press, Cambridge

Siniatchkin M, Groening K, Moehring J et al (2010) Neuronal networks in children with continuous spikes and waves during slow sleep. Brain 133:2798-2813. https://doi.org/10.1093/brain/awq183

Tassinari CA, Rubboli G (2019) Encephalopathy related to Status Epilepticus during slow Sleep: current concepts and 
future directions. Epileptic Disord 21(S1):82-87. https://doi. org/10.1684/epd.2019.1062

van den Munckhof B, Zwart AF, Weeke LC et al (2020) Perinatal thalamic injury: MRI predictors of electrical status epilepticus in sleep and long-term neurodevelopment. NeuroImage Clin 26:102227. https://doi.org/10.1016/j.nicl.2020.102227

Zuo S, Pan P, Li Q et al (2017) White matter injury and recovery after hypertensive intracerebral hemorrhage. Biomed Res Int 2017:111. https://doi.org/10.1155/2017/6138424
Publisher's Note Springer Nature remains neutral with regard to jurisdictional claims in published maps and institutional affiliations. 